\title{
Where is Psychiatry Headed?
}

\author{
Abraham Peled* \\ Clinical Associate Professor and Head of Department of Psychiatry, Rappaport Faculty of Medicine, Technion, Israel Institute of Technology, \\ Israel \\ *Corresponding author: Abraham Peled, Clinical Associate Professor and Head of Department of Psychiatry, Rappaport Faculty of \\ Medicine, Technion, Israel Institute of Technology, Haifa, Israel
}

\begin{tabular}{|c|c|}
\hline ARTICLE INFO & ABSTRACT \\
\hline Received: 幽 August 03, 2020 & Citation: Abraham Peled. Where is Psychiatry Headed?. Biomed J Sci \& Tech Res 29(3)- \\
\hline Published: 幽 August 12, 2020 & 2020. BJSTR. MS.ID.004813. \\
\hline
\end{tabular}

\section{Opinion}

In recent years we have witnessed two novel directions of development that promise a fascinating future for the world of psychiatry: the first encompasses technological innovations and the second includes scientific advances in the field of research called "neural computation". We live in a new technological world of sensors that are networked through the internet and are capable of measuring and tracking information and activities of the users. Daily activities of each and every one of us, reflect, among other things, our psychological states. Changes in user behavior can be detected, measured, and monitored and can be used to diagnose possible development of psychiatric disorders. Psychiatric diagnoses are based on signs and symptoms. Symptoms include the subject's complaints and signs reflect the examiner's observations. The observation may include appearance, facial expression, behavior (motor movements), spacial mobility, speech, judgement, and cognition. In the technological age, it is possible to extract a comprehensive observation of all of the above via the smartphone. Appearance and facial expressions are easily extracted from the camera on the smartphone; behavior and spacial mobility are measured with the GPS and analysis of the user's typed responses to various activities may indicate cognition. Symptoms, or patient's complaints, can be evaluated via questionnaires that the user is asked to complete, conveniently, using existing apps. Every psychiatric disorder has distinct characteristics. These features can be collected via the smartphone, stored, monitored, and analyzed so that an automatic continuous diagnostic process enables the psychiatrist to diagnose, monitor and even treat, remotely.

This can all be accomplished without the need for a physical visit to the clinic. Collection and statistical processing of the data naturally enable the development of accurate diagnostic measures, based on the characteristics of the complaints and observations of a large number of subjects. Once sufficient data are collected, artificial intelligence learning systems are activated to diagnose, detect, and monitor the patient's response to treatment, in a way that to date has not been possible. The introduction of intelligence systems in conjunction with a digitized sensor network promotes professional psychiatric efficiency that is several degrees higher than that of the individual psychiatrist. Digital psychiatry, of the type described here has a significant advantage in clinical practice, with emphasis on clinical surveillance of response to treatments. Conventional practice of physical follow-up visits in the clinic does not provide for a continuum of clinical evaluation, however, digital psychiatric monitoring allows for continuous clinical monitoring. When it comes to measuring exacerbation or treatment response, every change is revealed in real time in the continuous assessment, while waiting for a clinic-based assessment might take quite a long time and lead to missed or late detection of changes in patient status. Similarly, treatment response is affected by digital assessment. Continuous measurement of response to treatment beginning with treatment initiation saves the patient unnecessary and tedious waiting for a clinic appointment and reduces the possibility of delay in revealing a reaction related to effectiveness of the treatment.

The benefit to the patient is enormous, since it is possible to assess the efficacy in the initial stages of treatment and then decide whether to continue, adjust, or change pharmacotherapy. These benefits may potentially circumvent much pain and suffering for the patient. Alongside the numerous benefits of digital psychiatry, a principle innovation in comparison with conventional psychiatry is still lacking. The true psychiatric innovation will arrive when digital technology will unite with advances in the understanding of the 
brain - as a cause for psychiatric illnesses. Until recently, understanding of brain-related causes for mental disorders was lacking. That is why psychiatrists base their diagnoses on signs and symptoms rather than on laboratory tests and existing objective imaging, as in most fields of general medicine. This is also the reason that the psychiatric diagnostic system is not etiological (etiology means that the name of the disease is the cause of the disease. For example, intestinal obstruction is a medical diagnosis. The name of the disease indicates its location in the body - the intestine, and the pathology is the obstruction). A psychiatric diagnosis such as "depression" is not associated with a part of the body and does not describe a pathological mechanism. In the absence of knowledge of the cause of psychiatric disorders, the diagnoses remain descriptive - of the symptoms.

This may all change when the brain-related causes of psychiatric disorders are revealed. An evolving body of knowledge in brain research called "neural computation" will enable the understanding of the higher brain activities, often called mental or emotional activities, which function as complex "neural networks" of the brain. The brain is constructed as a complex system of neuronal networks (nerves). These networks spread throughout the brain, enabling organization of the brain with regard to emotions, thought, perception, consciousness, and cognition. In practice, the neural network activity supports the same higher mental functions that we attribute to the term "mental". When neuronal network organization is impaired for some reason, the higher mental functions are affected, with an impairment that might impede emotion, mood, perception, consciousness and cognition and reality testing. These are all characteristics of psychiatric disorders, which as noted are still described in the diagnostic framework of signs and symptoms. In order to describe the complex relationship between the organization of neural networks in the brain and mental functions and psychiatric disturbances, the metaphor of an orchestra can be used. Just as the orchestra creates music via coordination, synchronization, and complex timing among the musicians, so the neurons in the brain organize the networks and create the connectivity necessary to synchronize and coordinate higher mental and emotional functions. Without coordination among the musicians the music will be discordant.

The same goes for the brain: the more the neural networks are impaired in terms of coordination and synchronization, the more the mental activity will be impaired. If coordination among the musicians is lost, the music will become noise. In the brain, severe damage to organized neural network activity can result in the loss of higher mental abilities, as indeed is the case with severe psychiatric disorders. The field of neural computations has already offered quite a few insights regarding mental functions in psychiatry. One example is the brain's adaptation to stress states and the depressive response. For many years it has been known that anti-depressive agents stimulate the growth and development of nerve cells. The importance of the brain's adaptation to new, stressful, and painful situations is also well known. We also know that depression is associated with suffering and stress and nerve cell growth, in response to anti-depressive agents. However, until recently the relationship between these findings was not understood. With the help of neural computation, we are now able to understand that the growth and development of multiple nerve cells allows the brain to better adapt to situations of stress and suffering. In fact, the field of neural computation teaches us that the brain acts as an adaptation machine in that it predicts changes in the human environment and then adapts itself! The brain updates the internal representation of the external environment. The better the adjustment and adaptation of the brain to a person's changing life events, the better his or her stable mood is maintained. In contrast, impaired brain adaptation (due to impaired nerve cell growth) will be manifested as a reduction in the capacity of brain adaptation, leading to a decline in mood. This insight from the field of neural computation explains both the action of antidepressants and the importance of reducing stress in the treatment for depression. These insights pave the way for planning new therapeutic interventions not previously considered, based on the brain's role in nerve development and growth.

Confucius said: "The beginning of wisdom is to call things by their proper name". In the spirit of this ancient saying, the time has come to call psychiatric diseases by their proper names, the names of the cerebral mechanisms that cause them. By consolidating the fields of knowledge of neural computation and conventional psychiatry, a real revolution in the understanding and treatment of mental illness will soon be possible. In order to close the gap between the above insight and general medicine, and to understand the inherent therapeutic direction, the new approach to psychiatry can be compared to other fields of medicine, such as cardiology. Heart failure can be caused by a disturbance in the heart rhythm. The treatment will include insertion of a pacemaker to regulate the rhythm, in order to treat the symptoms and restore normal heart rate until the heart patient is cured. Similar to this example, psychiatric disorders can be compared to various forms of insufficient brain function caused by disorders of the neural networks in the brain. Development of a brain pacemaker (neuromodulation) will enable "repair" of the cerebral "rhythm disturbance" (that is disturbances in the neural network organization in the brain) and thus eliminate the symptoms and functional limitations.

The understanding that brain pacemakers will play an important role in curing psychiatric illnesses in the future raises the question - where do we stand today in terms of technology that allows for intervention and control of the brain? Indeed, brain intervention technology exists and is developing very rapidly, for example neurotechnology, neuromodulation, brain stimulation (electrical magnetic) and more. Direct electrical stimulation of the brain via implantation of electrodes directly into the cortex has been known for many years as a treatment for Parkinson's and a number of other brain diseases. Non-invasive technologies such as Transcranial Direct Current Stimulation (TDCS) and Transcranial Alternating Cur- 
rent Stimulation (tCAS) also facilitate the relief of numerous medical indications and quality of life issues. At the same time, in recent years, this technology has undergone vast improvements with the experience of Elon Musk (yes, the entrepreneur from Tesla and Space-X) with Neural ink and others to directly connect the brain to the computer. This is a familiar well-known field of brain-computer interfaces. Neural ink is currently developing tiny, delicate robots capable of transplanting 10,000 electrodes in the cortex in less than an hour. Because it is invasive, the technology of brain transplants can discourage patients and caregivers.

Thus, there are already even more innovative developments based on "neuronal dust". These are tiny (like dust) components of "nanotechnology" that can be dispersed in brain tissue through injection into the blood or spinal fluid. These particles can be activated externally by weak magnetic fields or weak ultrasound energy. When activated, these particles can activate and measure the neurons in their environment by sending a repetitive signal that is measured using appropriate sensors. Neuron dust - which both activates and measures brain activity, may well be the basis for the

ISSN: 2574-1241

DOI: $10.26717 /$ BJSTR.2020.29.004813

Abraham Peled. Biomed J Sci \& Tech Res

CC (i) This work is licensed under Creative Submission Link: https://biomedres.us/submit-manuscript.php future pacemaker, which requires a response-measuring circuit to stabilize and reorganize brain activity. Beyond that, this kind of penetrating technology will be more appropriate and safer for broad use, as needed. It is important to understand that the cure of psychiatric illness using remedial technology for brain efficiency does not have to end in the field of pathology. This technology opens the door to brain empowerment, which is the basis for success in the modern, competitive, and complex era. Everyone can actually benefit from enhancing and empowering their cognitive and emotional abilities. In summary, it is important to emphasize that psychiatric-digital measurement and monitoring and psychiatric diagnosis based on neural computation and neuromodulation technology are interrelated. In order to validate brain-based neural computational diagnosis, it is imperative to gather a mega data base, possible only through digital psychiatry. In order to activate an effective "brain pacemaker" it is vital to accurately diagnose the "disturbance of the brain rhythm" which is possible only via neural computation. There are currently quite advanced tools that enable rapid effective diagnosis alongside use of relatively simple non-invasive tools for various treatments.

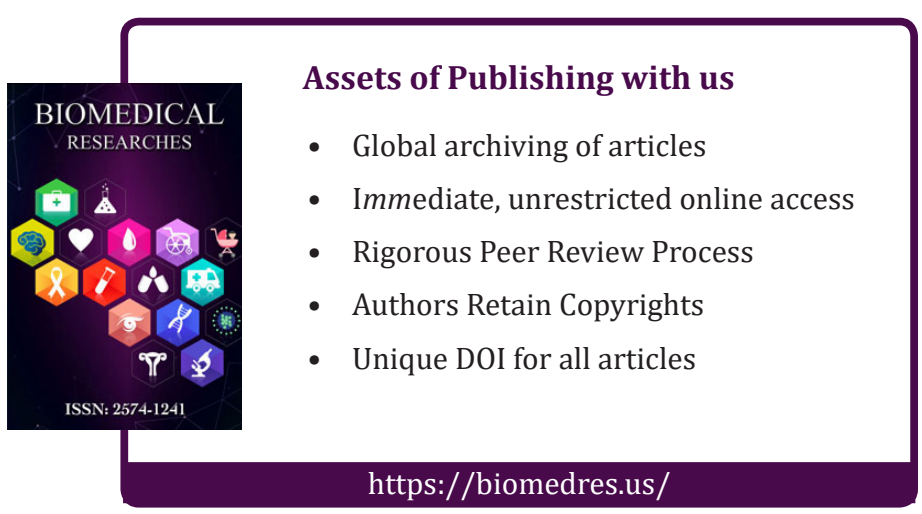

